\title{
EFFECT OF NITROGEN LEVEL, SEED MOISTURE CONTENT AND STORAGE PERIODS ON THE FUNGAL SEED DISCOLORATION IN SOME RICE CULTIVARS \\ EI-Wahsh, S. M. ${ }^{1}$; S. A. Fayzalla ${ }^{2}$ and A. A. Hassan ${ }^{1}$ \\ 1- Rice Pathol. Dept., Plant Pathol. Res. Inst., Agric. Res. Center, Egypt. 2- Plant Pathol. Dept. Fac. of Agric., Mansoura Univ., Egypt.
}

\begin{abstract}
Experiments were carried out at Rice Research and Training Center during 2004 growing season and storage period from October 2004 to March 2006. The study aimed to investigate the effect of grain storage periods with different levels of nitrogen fertilization $(0,23,46$ and $69 \mathrm{Kg} \mathrm{N} /$ fed.) or storage moisture content of 14 , 16,18 and $20 \%$ on discolored grains, fungal association and seed germination. The results showed that percentage of discolored grain and fungal association with rice grains increased by increasing storage periods regardless to the moisture content. On the other hand, seed germination decreased with the increase of storage period. Two types of bags were used to store the grains (striped plastic and jute sacks). The percentage of grain discoloration and associated fungi increased in striped plastic than in the jute sacks. The discoloration percentage and associated fungi on stored grains increased under 0 and 69 compared with 46 or 23 nitrogen levels. Giza 177 was the most affected cultivar with previous nitrogen levels, followed by Sakha 101, while, Giza 178 was the least one. Concerning the effect of rice grain moisture content with storage period on rice cultivars, data showed that the discoloration percentage increased with increasing of grain moisture content, while the germination percentage decreased. Giza177 and Sakha 104 were the most affected cultivars showing the highest discoloration percentage, associating fungi and the least germination. Alternaria spp, Bipolaris oryzae and Fusarium spp, were the most common fungi on grains before storage, while after 16 months of storage, the common fungi were Aspergillus spp and Penicillium spp. Presence of Rhizopus spp was the least one. In the greenhouse, eleven fungi were used for artificial inoculation on Giza 177. Bipolaris oryzae induced the highest grain discoloration (58.6\%) followed by Alternaria padwickii. and Curvularia lunata with 51.3 and $48.2 \%$, respectively. While Aspergillus spp and Penicillium spp. and un-inoculated pots showed the least grain discoloration with $2.2,1.9$ and $1.5 \%$, respectively.
\end{abstract}

\section{INTRODUCTION}

Rice (Oryza sativa L.) is one of the most important cereal crops in the world that is used in feeding more than half of the world's population (IRRI, 1997). The rice seed discoloration results in heavy losses to the crop (Das and Narain, 1988). The post-harvest treatments are important to maintain rice yield and quality. These treatments include temperature of drying, storage moisture content, storage conditions and storage duration (Danield et al 1988 \& Pearce et al 2001). Seed health refers primarily to the presence or absence of disease-causing organisms such as fungi, nematodes, bacteria, viruses and insects associated with seed (Fujisaka et al., 1993). The importance of seed quality in realizing full potential of a variety is well known. Seed vigor is recognized as an important seed quality parameter distinct from 
germinability (Seshu et al., 1988). Misra (1992) found that seed discoloration of rice (associated with several fungi) increased by increasing the levels of $\mathrm{N}$ fertilizers. El- Wahsh (1997) reported that seed discoloration was higher at zero nitrogen and high nitrogen $(80 \mathrm{~kg} / \mathrm{fed})$, but lower at $40 \mathrm{~kg} / \mathrm{fed}$. Pinciroli et al., (2004) found that the grain germination percentage decreased significantly with the higher doses of nitrogen. They added that germination was inhibited in variable proportion by different fungi with a range between $23.2 \%$ (in case of Epicoccum spp) and 100\% (in case of Bipolaris oryzae). Mallick and Nandi (1979) \& Shatta and Salem (1999) reported that Rice seeds stored on 12 to $21 \%$ moisture contents for up to 135 days in $70 \%$ relative humidity at $28 \pm 2^{\circ} \mathrm{C}$ increased invasion of grain by fungi and reduced germinability were proportional to the increase in grain moisture content and to increased time in storage. Also, at higher moisture content, the population of storage fungi, mainly species of Aspergillus and Penicillium increased with increasing storage period. Rahman and Mata (1998) found that seeds in storage carried both field and storage fungi. The most predominant field fungi were Alternaria padwickii, Bipolaris oryzae and Curvularia lunata and the predominant storage fungi were Aspergillus spp. and Penicillium spp. The incidence of storage fungi was higher in earthen containers than in gunny bags. Macedo et al (2002) evaluated the health quality of rice seeds stored in multifoliated paper, and polyethylene bags. There was no effect of bag type on seed health. The fungi found on the rice seeds were Pyricularia grisea, Bipolaris oryzae, Fusarium spp., Phoma spp., Curvularia spp., Cladosporium spp., Alternaria spp., Penicillium spp. and Aspergillus spp. The incidence of Penicillium spp. and Aspergillus spp. increased gradually during 12 months of the experiment. Sunaina et al (1988) observed gradual increase in seed discoloration through out year, but percentage germination was unaffected. Fusarium equiseti was the predominant of six species detected in the standing crop before storage. Aspergillus and Penicillium spp. were only recorded in samples of the standing crop after storage.

The objectives of the current study were to determine the effect of packaging materials, storage period with different moisture content and nitrogen fertilization rates on grain discoloration, seed viability and common fungi in rice seeds during storage.

\section{MATERIALS AND METHODS}

Effect of storage periods, nitrogen fertilization levels and type of bags on grain discoloration, seed viability and fungi occurring in rice seeds:-

Seed samples of Giza 177, Giza 178 and Sakha 101 rice cultivars were collected from verification experiments at two governorates i.e. Kafr Elsheikh and Dakahlia in 2004 season. The seeds were stored starting from October 2004 up to March 2006 at Rice Research \& Training Center (RRTC) laboratory at Sakha. Four levels of nitrogen (urea $46.5 \% \mathrm{~N}$ ), 0, 23, 46 and 69 $\mathrm{Kg}$ nitrogen/ fed were applied. Two thirds of the nitrogen dose was incorporated into the dry soil, while the last third was added thirty days after transplanting. A split-plot design with four replication were used. The rice 
cultivars were in main plots, where nitrogen levels were in sub-plots, the plot size was $4 \times 5 \mathrm{~m}$. Thirty day-old seedlings were transplanted at the spacing of $20 \times 20 \mathrm{~cm}$ with three plants/ hill. After harvest, the seeds were sun dried and adjusted to $14 \%$ moisture content (Gann hydromette G 86 Set). The seeds were stored in two types of bags, jute sacks and striped plastic under open shed storage for eighteen months. Seed samples were taken every six months of storage for testing by blotter methods (ISTA; 2004).

Effect of moisture content, storage periods of rice cvs. on grain discoloration, seed viability and fungi occurring in rice seeds :-

This experiment was conducted during 2004 growing season at Sakha Agricultural Research Station Farm. Five rice cultivars namely Giza 177, Giza 178, Giza 182, Sakha 101 and Sakha 104 were tested in a completed randomized block design (R-C-B-D) with four replications. The plot size was $4 \times 5 \mathrm{~m}$. Thirty day-old seedlings were transplanted in rows with three plants/ hill. The nitrogen fertilizer was used as urea $(46.5 \% \mathrm{~N})$ at 60 units $\mathrm{N} /$ fed. Two thirds of the nitrogen dose was incorporated into the dry soil, while the last third was added thirty days after transplanting. Just after harvest, the seed samples were collected and moisture content were adjusted by Gann hydromette G 86 Set to $14,16,18$ and $20 \%$ with sun drying. The samples were stored under open shed storage for sixteen months starting from October 2004 up to January 2006. Seed samples were taken every four month of storage to determine fungi associated with rice seeds, percentage of seed discoloration and seed viability.

\section{Technical assessment:-}

\section{A - Blotter Method:}

This method is used for detecting of fungi associated with rice seeds as suggested by ( ISTA 2004). In this experiment, the morphological characters of fungi, which formed the base for identification of different seed borne pathogens, were carefully observed directly over seeds using stereobinocular microscope. In this method, three layers of white blotters were Jointly soaked in sterilized water and placed in $9 \mathrm{~cm}$ diameter Petri dishes. Twenty five seeds, depending on their size, were placed per Petri plate at equal distance and 400 seeds were taken for each treatment. The plates were then incubated at $25^{\circ} \mathrm{C}+1$ under alternating cycles of $12 \mathrm{~h}$ near-ultra violet (NUV) light and $12 \mathrm{~h}$ darkness. Seeds were examined after 5-8 days under stereo-binocular microscope for the determination of fungi. The compound microscope was also used for confirmation of occurring fungi.

\section{B-Dry examination:}

One hundred seeds were examined visually under stero-binocular microscope to separate discolored seeds from healthy ones, (Khanzada and Khan 1987).

\section{Germination Test:}

400 seeds were incubated on two layers of blotting papers moistened with distilled water and placed in Petri dishes (25 seeds/plate ) at $28^{\circ} \mathrm{C} \pm 1$. The germination percentage was calculated after 5-8 days. 
Laboratory and greenhouse studies:-

Samples of discolored stored grain of different cultivars were used to isolate the most common fungi detected in this study. Among the isolated fungi, only eleven were selected for conducting pathogenicity test as presented in Table (10), by single inoculation under greenhouse conditions.

\section{Fungal Isolation:}

The discolored grains were surface sterilized by immersing in $0.5 \%$ sodium hypochlorite solution for 2 minutes, washed several times in sterilized distilled water and blotted between two sterilized filter papers, then transferred into Petri dishes containing filter paper. Sporulation occurred 48 hours after incubation in Petri dishes at $25^{\circ} \mathrm{C}$ and $100 \%$ relative humidity under continuous light fluorescent. Monoconidial cultures of the isolated fungi were obtained by the use of single spore method on $20 \%$ water agar (WA) for 24 hours, under $12 / 12 \mathrm{hr}$. dark and light at $25^{\circ} \mathrm{C}$ in the incubator. The germinated single conidia were picked and transferred to be grown and multiplied on PDA medium (200g potato, $20 \mathrm{~g}$ dextrose and $20 \mathrm{~g}$ agar $/ 1000$ $\mathrm{ml}$ distilled water) and incubated under fluorescent light for 10 days at $25^{\circ} \mathrm{C}$, then the spores were harvested. (at density at least 25 spores / microscopic field).

Pathogenicity test:

Giza 177 rice cultivar (the most sensitive for grain discoloration) was seeded in plastic pots ( $30 \mathrm{~cm}$ diameter ), as three hills (five grains / hill ) per pot. The pots were kept in the greenhouse at $25-30^{\circ} \mathrm{C}$ and fertilized with urea $46.5 \% \mathrm{~N}$, for three times at of $5 \mathrm{~g} /$ pot. Artificial inoculation with $100 \mathrm{ml}$ spore suspension of each of the tested fungi adjusted to $5 \times 10^{4}$ spores $/ \mathrm{ml}$ were sprayed at early heading stage. Electerical spray gun (atomizer) was used for inoculation of four replicates for each fungus. While, $100 \mathrm{ml}$ of distilled water was sprayed as check. $2.5 \mathrm{~g} / \mathrm{l}$ of gelatin were added to spore suspension inoculums to enhance infection (Bastiaan, 1993). The inoculated plants were held in a moist chamber with $90 \%$ R.H at least and $25-28^{\circ} \mathrm{C}$ for 48 hours, then moved to the greenhouse conditions. At the late of maturity stage, the crop was harvested and the discolored grain percentage and unfilled grains were determined.

\section{RESULTS AND DISCUSSION}

Effect of storage periods, nitrogen fertilization levels and type of bags on grain discoloration, seed viability and fungi occurring in rice seeds :-

Data in Table (1) indicated that the percentage of discolored rice grains was higher in the plots without nitrogen application (zero) or higher (69 $\mathrm{Kg} \mathrm{N} / \mathrm{fed}$ ) nitrogen rate than in that with 23 and $46 \mathrm{Kg} \mathrm{N} / \mathrm{fed}$ for all cultivars. The lowest discolored grains percentage was obtained under $46 \mathrm{~kg} \mathrm{~N} / \mathrm{fed}$. followed by $23 \mathrm{~kg} \mathrm{~N} / \mathrm{fed}$. Giza 177 had the highest seed discoloration followed by Sakha 101, while Giza 178 had the lowest percentage. The discolored grain increased gradually by increasing storage periods for all cultivars. The highest seed discoloration appeared after eighteen months of storage. These results are in agreement with Misra et al (1992) \& El- Wahsh (1997) and 
Pinciroli et al (2004) who reported that the discoloration of grains was different from cultivar to another. Also, seed discoloration increased with low or high nitrogen fertilizer. Seed discoloration was higher with rice grains stored in striped plastic than in jute sacks for all rice cultivars. On the other hand, the seed germination decreased by increasing storage period. Giza 178 gave the highest germination compared with Sakha 101 or Giza 177 (Table 2). Seeds stored in jute sacks gave higher germination than those stored in striped plastic especially with increasing storage period, (Table 2). [Rao (1992) and Muangkaw et al. (2005) reported that the rice germinibility and discoloration differed according to type of storage]. Data in Tables (3\&4) show that the occurrence of most fungi increased gradually with increasing storage period (from 6 to 18 months). The highest increase was recorded with Aspergillus spp and Penicillium spp. The occurrence of Bipolaris oryzae on Giza $177 \mathrm{cv}$. decreased gradually from 14.9 to 5.1 in striped plastic bags and from 14.9 to 7.3 with jute sacks. The highest fungal occurrence was recorded on Giza 177 followed by Sakha 101 and Giza 178. Storage in jute sacks reduced the occurrence of rice grains associated fungi compared with striped plastic at both Kafr El-Sheikh and El-Dakahlia governorates. For example, Alternaria spp. on Giza 177 rice cv. increased from $18.8 \%$ prior to storage to $24.7 \%$ after 18 months storage in jute sacks compared with 18.8 $\%$ to 30.7 for the same treatment in striped plastic at Kafr El-Sheikh (Table3). The same trend was observed at El- Dakahlia governorate (Table 4). Results in Tables (5\&6) showed that Bipolaris oryzae was higher on Giza 177 than on Sakha 101 and Giza 178 as affected by 0 and $69 \mathrm{Kg} \mathrm{N} / \mathrm{fed}$. at Kafr El-Sheikh and El-Dakahlia governorates. This may be due to the higher susceptibility of Giza 177 than the two other cultivars. Also, lower and higher nitrogen fertilizer rates are known to increase brown spot infection caused by Bipolaris oryzae as indicated by Shatta and Salem (1999). Also, Mia and Safeeulla (1998) found that viability of the $B$.oryzae gradually declined when seed with high moisture content $(16 \%)$ were stored at $25 \pm 1^{\circ} \mathrm{C}$. Low temperature $\left(15 \pm 1^{\circ} \mathrm{C}\right)$ storage counteracted the high moisture effect.

Generally, the higher percentage of occurrence of Alternaria spp. and Fusarium spp. were found on all cultivars at all nitrogen rates and the two types of bags as field infecting fungi than Nigrospora oryzae and Curvularia lunata in the tow governorates, (Tables 5\&6). This may be due to the high effect of Alternaria spp. and Fusarium spp. on rice grain discoloration in addition to Biploaris oryzae than Nigrospora oryzae and Curvularia lunta. However, both Aspergillus spp. and Penicillium spp. were more common as storage fungi than Rhizopus spp. especially at 0 and $69 \mathrm{Kg} \mathrm{N}$./fed on the tested cultivars (Table 5\&6). 
El-Wahsh, S. M. et al.

1

2002 
J. Agric. Sci. Mansoura Univ., 33 (3), March, 2008

2

2003 
El-Wahsh, S. M. et al.

Table (3): Mean percentage of common fungi occurring on seeds of rice cultivars stored for different periods in two types of bags regardless of nitrogen fertilization at Kafr-El-Sheikh governorate.

\begin{tabular}{|c|c|c|c|c|c|c|c|c|c|}
\hline \multirow[t]{2}{*}{ Cultivar } & \multirow[t]{2}{*}{ Fungi } & \multicolumn{4}{|c|}{$\begin{array}{l}\text { Striped plastic / storage } \\
\text { period (month) }\end{array}$} & \multicolumn{4}{|c|}{$\begin{array}{l}\text { Jute sacks / storage } \\
\text { period (month) }\end{array}$} \\
\hline & & $0^{*}$ & 6 & 12 & 18 & $0^{*}$ & 6 & 12 & 18 \\
\hline \multirow{8}{*}{$\frac{N}{\frac{N}{N}}$} & Bipolaris oryzae & 14.9 & 18 & 11.8 & 5.1 & 14.9 & 16.9 & 13.1 & 7.3 \\
\hline & Alternaria spp & 18.8 & 18.4 & 23.3 & 30.7 & 18.8 & 16.5 & 21.7 & 24.7 \\
\hline & Fusarium spp & 11.6 & 15 & 21.3 & 28.3 & 11.6 & 13.5 & 16.3 & 22.7 \\
\hline & Nigrospora oryzae & 5.5 & 6.8 & 8.6 & 9.5 & 5.5 & 6.6 & 8.2 & 9.3 \\
\hline & Curvularia Iunata & 4.1 & 4.9 & 5.8 & 6.5 & 4.1 & 4.8 & 5.6 & 6.1 \\
\hline & Aspergillus spp & 4.3 & 13.7 & 27.3 & 39.5 & 4.3 & 10.7 & 28.0 & 30.0 \\
\hline & Penicillium spp & 3.3 & 14.5 & 26 & 32.7 & 3.3 & 12.5 & 22.7 & 31.0 \\
\hline & Rihzopus spp & 0.5 & 3.3 & 5.3 & 7.5 & 0.5 & 1.3 & 1.7 & 6.0 \\
\hline \multirow{8}{*}{$\begin{array}{l}\stackrel{\infty}{\frac{\infty}{N}} \\
\frac{\mathbb{N}}{\mathbb{N}}\end{array}$} & Bipolaris oryzae & 8.8 & 11.3 & 5.4 & 1.5 & 8.8 & 10.4 & 6.5 & 2.1 \\
\hline & Alternaria spp & 8.7 & 11.1 & 14.7 & 18.7 & 8.7 & 10.5 & 12.7 & 15.3 \\
\hline & Fusarium spp & 6.3 & 8.9 & 10 & 14.7 & 6.3 & 6.8 & 8.1 & 11.7 \\
\hline & Nigrospora oryzae & 3.1 & 3.2 & 4.1 & 4.8 & 3.1 & 3.2 & 3.6 & 4.4 \\
\hline & Curvularia Iunata & 1.6 & 2.3 & 3 & 3.7 & 1.6 & 1.9 & 2.3 & 2.9 \\
\hline & Aspergillus spp & 4.3 & 10.3 & 21.0 & 27.0 & 4.3 & 8.3 & 17.7 & 23.3 \\
\hline & Penicillium spp & 3.1 & 7.7 & 18.5 & 24.5 & 3.1 & 6.3 & 15.7 & 20.7 \\
\hline & Rihzopus spp & 0.3 & 1.0 & 2.7 & 4.7 & 0.3 & 1.0 & 1.7 & 3.3 \\
\hline \multirow{8}{*}{ 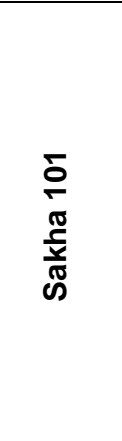 } & Bipolaris oryzae & 12.6 & 14.7 & 8.9 & 2.2 & 12.7 & 13.6 & 9.7 & 4.6 \\
\hline & Alternaria spp & 12.6 & 15.7 & 20.0 & 23.0 & 12.6 & 14.5 & 17.5 & 20.3 \\
\hline & Fusarium spp & 8.1 & 11.0 & 14.7 & 20.0 & 8.1 & 8.7 & 11.3 & 17.5 \\
\hline & Nigrospora oryzae & 2.6 & 3.6 & 4.5 & 5.7 & 2.6 & 3.2 & 4.0 & 4.7 \\
\hline & Curvularia lunata & 2.4 & 3.2 & 3.9 & 5.1 & 2.4 & 2.5 & 3.8 & 4.5 \\
\hline & Aspergillus spp & 4.3 & 12.7 & 23 & 28.7 & 4.3 & 9.2 & 18.7 & 24.5 \\
\hline & Penicillium spp & 4.7 & 11.3 & 21.3 & 26.7 & 4.7 & 8.5 & 17.7 & 23.5 \\
\hline & Rihzopus spp & 0.5 & 1.5 & 1.7 & 2.3 & 0.5 & 1.0 & 1.7 & 2.3 \\
\hline & L.S.D $5 \%$ & \multicolumn{4}{|c|}{1.45} & \multicolumn{4}{|c|}{1.54} \\
\hline
\end{tabular}

Prior to storage 
Table (4): Mean percentage of common fungi occurring on seeds of rice cultivars stored for different periods in two types of bags regardless of nitrogen fertilization at El-Dakahlia governorate.

\begin{tabular}{|c|c|c|c|c|c|c|c|c|c|}
\hline \multirow[t]{2}{*}{ Cultivar } & \multirow[t]{2}{*}{ Fungi } & \multicolumn{4}{|c|}{$\begin{array}{l}\text { Striped plastic / storage } \\
\text { period (month) }\end{array}$} & \multicolumn{4}{|c|}{$\begin{array}{l}\text { Jute sacks / storage period } \\
\text { (month) }\end{array}$} \\
\hline & & $0^{*}$ & 6 & 12 & 18 & $0^{*}$ & 6 & 12 & 18 \\
\hline \multirow{8}{*}{$\begin{array}{l}\frac{N}{\mathbb{N}} \\
\frac{\mathbb{N}}{\mathbb{N}}\end{array}$} & Bipolaris oryzae & 14.7 & 16.9 & 10.8 & 3 & 14.7 & 16.1 & 12.6 & 4.0 \\
\hline & Alternaria spp & 13.8 & 17.2 & 22.7 & 27 & 13.8 & 15.7 & 20.0 & 23 \\
\hline & Fusarium spp & 11.3 & 13.5 & 18.5 & 25.7 & 11.3 & 12.3 & 14.8 & 21.2 \\
\hline & Nigrospora oryzae & 3.9 & 5.7 & 7.3 & 8.4 & 3.9 & 5.3 & 6.5 & 7.8 \\
\hline & Curvularia Iunata & 3.1 & 3.8 & 4.5 & 5.6 & 3.1 & 3.7 & 4.2 & 5.1 \\
\hline & Aspergillus spp & 4.3 & 10.5 & 23.7 & 34.7 & 4.3 & 8.3 & 19.7 & 26.7 \\
\hline & Penicillium spp & 3.7 & 12.3 & 23.5 & 33.3 & 3.7 & 9.5 & 20.0 & 26 \\
\hline & Rihzopus spp & 0.0 & 2.3 & 3.7 & 9.3 & 0.0 & 1.3 & 3.3 & 6.7 \\
\hline \multirow{8}{*}{$\frac{\infty}{\frac{\infty}{N}}$} & Bipolaris oryzae & 10.1 & 12.2 & 7.3 & 1.7 & 10.1 & 11.2 & 7.3 & 2.3 \\
\hline & Alternaria spp & 12.0 & 13.4 & 16.5 & 21.0 & 12 & 13 & 15.0 & 17.7 \\
\hline & Fusarium spp & 6.9 & 9.7 & 11.5 & 16.7 & 6.9 & 7.5 & 9.0 & 14.0 \\
\hline & Nigrospora oryzae & 2.3 & 3.6 & 5.1 & 5.7 & 2.3 & 2.8 & 4.0 & 4.9 \\
\hline & Curvularia lunata & 1.9 & 2.8 & 3.6 & 4.6 & 1.9 & 2.3 & 2.8 & 3.7 \\
\hline & Aspergillus spp & 12.1 & 11.3 & 22.7 & 29.5 & 12.1 & 8.3 & 16.7 & 21 \\
\hline & Penicillium spp & 3.7 & 9.0 & 19.5 & 26.5 & 3.7 & 7.7 & 16.7 & 20 \\
\hline & Rihzopus spp & 0.5 & 1.0 & 2.0 & 3.0 & 0.5 & 0.3 & 1.0 & 1.7 \\
\hline \multirow{8}{*}{ 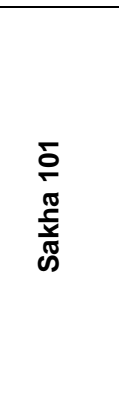 } & Bipolaris oryzae & 14.3 & 16.5 & 11.4 & 3.1 & 14.3 & 15.4 & 12.2 & 4.1 \\
\hline & Alternaria spp & 14.6 & 17.3 & 23.5 & 26 & 14.6 & 17 & 19.7 & 22.7 \\
\hline & Fusarium spp & 9.0 & 11.6 & 16.7 & 23.7 & 9.0 & 10.7 & 13.5 & 20.3 \\
\hline & Nigrospora oryzae & 3.4 & 4.2 & 5.9 & 6.8 & 3.4 & 4.0 & 4.8 & 5.7 \\
\hline & Curvularia Iunata & 2.7 & 3.6 & 4.9 & 6.3 & 2.7 & 3.5 & 4.2 & 5.3 \\
\hline & Aspergillus spp & 4.7 & 10.5 & 22.3 & 28.5 & 4.7 & 8.5 & 18.4 & 26.5 \\
\hline & Penicillium spp & 3.7 & 9.7 & 20.7 & 29 & 3.7 & 8.0 & 16.0 & 22.3 \\
\hline & Rihzopus spp & 0.5 & 3.0 & 5.0 & 7.3 & 0.5 & 2.3 & 3.5 & 6.3 \\
\hline & L.S.D $5 \%$ & \multicolumn{4}{|c|}{1.11} & \multicolumn{4}{|c|}{1.09} \\
\hline
\end{tabular}


El-Wahsh, S. M. et al.

Table (5): Mean percentage of common fungi occurring on rice seeds stored (two types of bags) for18 months collected from rice cultivars grown under different nitrogen levels regardless of storage period at Kafr El-Shiekh governorate

\begin{tabular}{|c|c|c|c|c|c|c|c|c|c|}
\hline \multirow[t]{2}{*}{ Cultivar } & \multirow[t]{2}{*}{ Fungi } & \multicolumn{4}{|c|}{$\begin{array}{c}\text { Striped plastic/Nitrogen } \\
\text { level (kg/fed) }\end{array}$} & \multicolumn{4}{|c|}{$\begin{array}{c}\text { Jute sacks/ Nitrogen level } \\
(\mathrm{kg} / \mathrm{fed})\end{array}$} \\
\hline & & 0 & 23 & 46 & 69 & 0 & 23 & 46 & 69 \\
\hline \multirow{8}{*}{$\frac{N}{\frac{N}{N}}$} & Bipolaris oryzae & 18.8 & 9.3 & 7.6 & 14.1 & 19.6 & 9.7 & 8.1 & 14.7 \\
\hline & Alternaria spp & 23.4 & 21.3 & 19.7 & 26.8 & 19.7 & 18.7 & 17.0 & 26.3 \\
\hline & Fusarium spp & 19.3 & 17.5 & 15.7 & 23.5 & 16 & 15.5 & 12.5 & 20.5 \\
\hline & Nigrospora oryzae & 6.8 & 7.7 & 7.7 & 8.2 & 6.7 & 7.5 & 7.4 & 7.5 \\
\hline & Curvularia Iunata & 4.2 & 5.4 & 5.1 & 6.7 & 4.0 & 5.3 & 5.2 & 6.2 \\
\hline & Aspergillus spp & 21.8 & 19.0 & 17.2 & 26.2 & 18.0 & 20.2 & 14.5 & 22.8 \\
\hline & Penicillium spp & 19.3 & 17.3 & 16.0 & 23.0 & 16.8 & 15.1 & 15.0 & 22.0 \\
\hline & Rihzopus spp & 3.3 & 3.7 & 3.3 & 6.3 & 2.0 & 2.0 & 2.0 & 3.5 \\
\hline \multirow{8}{*}{$\begin{array}{l}\frac{\infty}{N} \\
\frac{\mathbb{N}}{\mathbb{N}}\end{array}$} & Bipolaris oryzae & 9.6 & 5.4 & 4.3 & 7.4 & 9.7 & 5.4 & 4.5 & 8.2 \\
\hline & Alternaria spp & 13.7 & 11.5 & 10 & 18.2 & 12.0 & 10.7 & 8.5 & 16.0 \\
\hline & Fusarium spp & 10.1 & 8.8 & 8.3 & 11.7 & 8.1 & 7.4 & 6.8 & 10.7 \\
\hline & Nigrospora oryzae & 3.6 & 3.6 & 2.8 & 5.1 & 3.2 & 3.5 & 2.8 & 4.7 \\
\hline & Curvularia lunata & 2.5 & 1.9 & 2.1 & 3.8 & 2.1 & 1.7 & 1.9 & 3.2 \\
\hline & Aspergillus spp & 15.8 & 13.8 & 13.8 & 19.2 & 13.8 & 11.6 & 11.8 & 16.4 \\
\hline & Penicillium spp & 13.2 & 12.0 & 12.0 & 16.6 & 11.0 & 10.5 & 10.5 & 13.8 \\
\hline & Rihzopus spp & 0.5 & 2.7 & 1.0 & 4.7 & 0.3 & 1.3 & 0.7 & 2.7 \\
\hline \multirow{8}{*}{$\begin{array}{l}\bar{\sigma} \\
\text { o } \\
\frac{\pi}{5} \\
\text { ஸे }\end{array}$} & Bipolaris oryzae & 14.3 & 6.7 & 6.1 & 11.2 & 14.7 & 6.9 & 6.1 & 11.2 \\
\hline & Alternaria spp & 17.8 & 16 & 14 & 23.6 & 16.1 & 14.3 & 12.5 & 22.1 \\
\hline & Fusarium spp & 13.5 & 12.5 & 10.8 & 16.9 & 10.7 & 10.7 & 9.6 & 14.5 \\
\hline & Nigrospora oryzae & 4.1 & 3.9 & 3.6 & 4.8 & 3.5 & 3.5 & 3.2 & 4.5 \\
\hline & Curvularia lunata & 3.3 & 3.9 & 2.7 & 4.6 & 2.7 & 3.6 & 2.4 & 4.2 \\
\hline & Aspergillus spp & 18.0 & 15.3 & 14.8 & 20.5 & 14.2 & 12.8 & 12.5 & 17.2 \\
\hline & Penicillium spp & 16.3 & 14.5 & 13.9 & 19.3 & 13.3 & 12.3 & 11.9 & 17.0 \\
\hline & Rihzopus spp & 1.5 & 2.0 & 0.0 & 2.5 & 2.0 & 0.7 & 0.5 & 2.0 \\
\hline & L.S.D $5 \%$ & \multicolumn{4}{|c|}{1.73} & \multicolumn{4}{|c|}{1.18} \\
\hline
\end{tabular}

Values in the table are the mean of four examinations at 6-month period 
Table (6): Mean percentage of common fungi occurring on rice seeds stored (two types of bags) for 18 months collected from rice cultivars grown under different nitrogen levels regardless of storage period at El-Dakahlia governorate.

\begin{tabular}{|c|c|c|c|c|c|c|c|c|c|}
\hline \multirow[t]{2}{*}{ Cultivar } & \multirow[t]{2}{*}{ Fungi } & \multicolumn{4}{|c|}{$\begin{array}{c}\text { Striped plastic/Nitrogen } \\
\text { level }(\mathrm{kg} / \mathrm{fed})\end{array}$} & \multicolumn{4}{|c|}{$\begin{array}{c}\text { Jute sacks/ Nitrogen level } \\
\text { (kg/fed) }\end{array}$} \\
\hline & & 0 & 23 & 46 & 69 & 0 & 23 & 46 & 69 \\
\hline \multirow{8}{*}{$\frac{N}{\frac{N}{N}}$} & Bipolaris oryzae & 16.6 & 8.4 & 6.6 & 13.8 & 17.1 & 8.9 & 7.2 & 14.3 \\
\hline & Alternaria spp & 19.9 & 18.6 & 17.5 & 24.8 & 17.5 & 16.6 & 15.3 & 23.3 \\
\hline & \begin{tabular}{|l|} 
Fusarium spp \\
\end{tabular} & 18.1 & 15.5 & 14.5 & 21.0 & 14.7 & 13.8 & 12.5 & 18.5 \\
\hline & Nigrospora oryzae & 5.7 & 6.4 & 6.2 & 7.2 & 5.3 & 6.0 & 5.7 & 6.6 \\
\hline & Curvularia Iunata & 2.9 & 4.5 & 4.3 & 5.4 & 2.5 & 4.3 & 4.0 & 5.2 \\
\hline & Aspergillus spp & 18.5 & 16.8 & 15.5 & 22.4 & 14.8 & 14.3 & 11.0 & 17.4 \\
\hline & Penicillium spp & 18.0 & 16.8 & 16.2 & 21.8 & 14.2 & 13.7 & 13.5 & 17.8 \\
\hline & Rihzopus spp & 3.7 & 3.0 & 3.3 & 5.3 & 3.0 & 2.0 & 2.0 & 4.3 \\
\hline \multirow{8}{*}{ 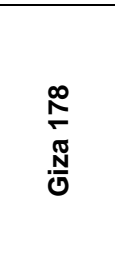 } & Bipolaris oryzae & 10.1 & 6.6 & 5.5 & 9.1 & 10.6 & 6.1 & 5.1 & 9.1 \\
\hline & Alternaria spp & 15.0 & 14.6 & 12.8 & 20.5 & 13.5 & 13.5 & 12 & 18.7 \\
\hline & Fusarium spp & 11.0 & 10 & 9.5 & 14.5 & 9.3 & 9.3 & 7.7 & 12 \\
\hline & Nigrospora oryzae & 4.2 & 3.7 & 2.8 & 5.8 & 4 & 2.5 & 1.8 & 5.6 \\
\hline & Curvularia Iunata & 2.9 & 3.1 & 2.5 & 4.5 & 2.1 & 3.2 & 1.9 & 3.6 \\
\hline & Aspergillus spp & 17.0 & 15.2 & 15.0 & 20.8 & 12.2 & 11.5 & 11.2 & 9.5 \\
\hline & Penicillium spp & 14.2 & 13.0 & 12.8 & 18.8 & 11.2 & 11.2 & 10.8 & 15.0 \\
\hline & Rihzopus spp & 2.0 & 1.5 & 0.3 & 2.3 & 1.3 & 0.7 & 0.3 & 1.7 \\
\hline \multirow{8}{*}{ 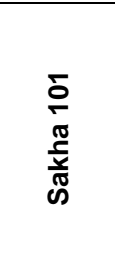 } & Bipolaris oryzae & 16.3 & 8.5 & 7.6 & 12.7 & 16.3 & 8.8 & 7.7 & 13.1 \\
\hline & Alternaria spp & 20.0 & 18.3 & 17.0 & 26.1 & 17.5 & 16.7 & 15.3 & 24.6 \\
\hline & Fusarium spp & 15.6 & 14.3 & 12.7 & 18.5 & 13.5 & 12.7 & 11 & 16.3 \\
\hline & Nigrospora oryzae & 4.6 & 4.9 & 5.2 & 6.2 & 4 & 4.3 & 4.4 & 5.5 \\
\hline & Curvularia Iunata & 4.0 & 4.4 & 3.4 & 5.7 & 3.5 & 3.9 & 2.8 & 5.5 \\
\hline & Aspergillus spp & 17.2 & 15.0 & 14.2 & 19.5 & 14.5 & 13.3 & 13.0 & 17.2 \\
\hline & Penicillium spp & 16.5 & 14.0 & 13.8 & 19.0 & 12.0 & 10.8 & 10.8 & 16.5 \\
\hline & Rihzopus spp & 3.7 & 3.3 & 3.0 & 5.7 & 3.3 & 2.3 & 1.7 & 5.3 \\
\hline \multicolumn{2}{|r|}{ L.S.D $5 \%$} & \multicolumn{4}{|c|}{1.58} & \multicolumn{4}{|c|}{0.55} \\
\hline
\end{tabular}

Values in the table are the mean of four examinations at 6-month period

Effect of grain moisture content at harvest on seed discoloration and invasion by storage fungi in rice cultivars:-

Results in Table (7) indicated that percentage of discolored grains slightly increased on the five tested cultivars when the grain moisture content was at its lower level (14\%). The increase was from 20.6 to $23.4 \%$ in Giza 177; from 10.0 to $20.0 \%$; 12.0 to $23.0 \% ; 21.0$ to $23.8 \%$ and 6.0 to $8.8 \%$ in Giza 178, Sakha 101, Sakhs 104 and Giza 182 prior to storage and after 16 months storage respectively. However, the increase in grain moisture content from $14-16 \%$ to $18-20 \%$ was accompanied with high increase in grain discoloration for all cultivars. The increase was from 22.3 to $83.0 \%$; 12.5 to $75.0 \% ; 13.2$ to $80.0 \%$; 22.3 to $85.0 \%$ and 7.2 to $70.1 \%$ for Giza 177 , Giza 178, Sakha 101, Sakha 104 and Giza 182 prior to storage and after 16 months storage, respectively (Table 7). This indicates the significant role of keeping the grain moisture content at $14 \%$ or lower to avoid higher percentages of grain discoloration especially with increasing periods of storage as indicated by Mallick and Nandi (1979) \& Shatta and Salem (1999) and Muangkaw et al (2005) who reported that discolored grains and fungi increased by increase of either moisture content in seeds stored or storage period. 
El-Wahsh, S. M. et al.

Table (7): Percentage of discolored rice grains of different rice cultivars as affected by storage period and levels of moisture content.

\begin{tabular}{|c|c|c|c|c|c|c|}
\hline \multirow{2}{*}{ Cultivar } & \multirow{2}{*}{$\begin{array}{l}\text { Moisture } \\
\text { content \% }\end{array}$} & \multicolumn{5}{|c|}{ Storage period (month) } \\
\hline & & $0^{*}$ & 4 & 8 & 12 & 16 \\
\hline \multirow{4}{*}{ Giza 177} & 14 & 20.60 & 21.10 & 21.20 & 23.10 & 23.40 \\
\hline & 16 & 20.60 & 23.00 & 26.00 & 30.00 & 48.00 \\
\hline & 18 & 22.10 & 25.00 & 28.00 & 68.00 & 80.00 \\
\hline & 20 & 22.30 & 30.00 & 35.00 & 72.00 & 83.00 \\
\hline \multirow{4}{*}{ Giza 178} & 14 & 10.00 & 10.10 & 14.00 & 16.00 & 20.00 \\
\hline & 16 & 10.10 & 11.00 & 16.00 & 20.00 & 35.00 \\
\hline & 18 & 12.30 & 18.10 & 50.00 & 63.00 & 72.00 \\
\hline & 20 & 12.50 & 23.60 & 60.00 & 68.00 & 75.00 \\
\hline \multirow{4}{*}{ Sakha 101} & 14 & 12.00 & 12.20 & 15.00 & 18.00 & 23.00 \\
\hline & 16 & 12.00 & 13.10 & 20.00 & 26.00 & 40.00 \\
\hline & 18 & 13.10 & 22.40 & 55.00 & 60.00 & 75.00 \\
\hline & 20 & 13.20 & 26.50 & 65.00 & 70.00 & 80.00 \\
\hline \multirow{4}{*}{ Sakha 104} & 14 & 21.00 & 21.70 & 21.20 & 23.70 & 23.80 \\
\hline & 16 & 21.10 & 23.60 & 25.10 & 43.00 & 55.00 \\
\hline & 18 & 22.10 & 26.00 & 31.00 & 60.00 & 80.00 \\
\hline & 20 & 22.30 & 31.10 & 35.00 & 74.00 & 85.00 \\
\hline \multirow{4}{*}{ Giza 182} & 14 & 6.00 & 6.10 & 6.60 & 7.80 & 8.80 \\
\hline & 16 & 6.20 & 7.90 & 9.10 & 9.80 & 12.30 \\
\hline & 18 & 7.00 & 17.60 & 23.10 & 48.10 & 66.90 \\
\hline & 20 & 7.20 & 19.10 & 29.90 & 62.30 & 70.10 \\
\hline L.S.D 5\% & & \multicolumn{5}{|c|}{3.36} \\
\hline
\end{tabular}

"Prior to storage.

Seed germination percentage as affected by moisture content (14$20 \%$ ) and storage periods (4-16 months) are presented in Table (8). At $14 \%$ grain moisture content, germination percentages decreased from $93.2 \%$ after 4 months storage to $81.0 \%$ after 16 months storage for Giza 177 rice cultivar. For other cvs., the reduction was from 94 to $88 \%$; 93.4 to $86 \%$; 92.6 to $81.0 \%$ and 97.4 to 90.0 for Giza 178, Sakha 101, Sakha 104 and Giza 182 , respectively. The increase in grain moisture content to $18 \%$ or higher showed severe reduction in germination percentages of the five tested cultivars. For example, germination percentage was reduced from $8 \%$ after 4 month storage to $0 \%$ (no germination) after 8 month storage (or more) for Giza 177 rice cv. (Table 8). The same trend was observed with other cultivars indicating the importance of keeping grain moisture content at $14 \%$ or lower to avoid seed deterioration especially with longer storage periods. There results are in accordance with those reported by Mallick and Nandi (1979) \& Shatta and Salem (1999) who reported that loss of germinability were proportional to the increase in grain moisture content and to increased time of storage. 
J. Agric. Sci. Mansoura Univ., 33 (3), March, 2008

Table (8): Percentage of seed germination of different rice cultivars as affected by storage period and levels of moisture content.

\begin{tabular}{|c|c|c|c|c|c|}
\hline \multirow{2}{*}{ Cultivar } & \multirow{2}{*}{$\begin{array}{l}\text { Moisture } \\
\text { content \% }\end{array}$} & \multicolumn{4}{|c|}{ Storage period (month) } \\
\hline & & 4 & 8 & 12 & 16 \\
\hline \multirow{4}{*}{ Giza 177} & 14 & 93.20 & 93.00 & 90.00 & 81.00 \\
\hline & 16 & 85.00 & 65.00 & 31.10 & 12.00 \\
\hline & 18 & 8.00 & 0.00 & 0.00 & 0.00 \\
\hline & 20 & 0.00 & 0.00 & 0.00 & 0.00 \\
\hline \multirow{4}{*}{ Giza 178} & 14 & 94.00 & 93.90 & 91.00 & 88.00 \\
\hline & 16 & 88.00 & 81.00 & 71.00 & 35.00 \\
\hline & 18 & 18.00 & 0.00 & 0.00 & 0.00 \\
\hline & 20 & 2.00 & 0.00 & 0.00 & 0.00 \\
\hline \multirow{4}{*}{ Sakha 101} & 14 & 93.40 & 93.00 & 90.10 & 86.00 \\
\hline & 16 & 87.00 & 70.00 & 59.00 & 30.00 \\
\hline & 18 & 12.00 & 0.00 & 0.00 & 0.00 \\
\hline & 20 & 1.00 & 0.00 & 0.00 & 0.00 \\
\hline \multirow{4}{*}{ Sakha 104} & 14 & 92.60 & 92.00 & 90.00 & 81.00 \\
\hline & 16 & 82.00 & 53.00 & 29.00 & 10.00 \\
\hline & 18 & 7.00 & 0.00 & 0.00 & 0.00 \\
\hline & 20 & 0.00 & 0.00 & 0.00 & 0.00 \\
\hline \multirow{4}{*}{ Giza 182} & 14 & 97.40 & 97.20 & 95.00 & 90.00 \\
\hline & 16 & 94.00 & 87.00 & 84.00 & 66.00 \\
\hline & 18 & 25.00 & 1.00 & 0.00 & 0.00 \\
\hline & 20 & 10.00 & 0.00 & 0.00 & 0.00 \\
\hline \multicolumn{2}{|c|}{ L.S.D 5\% } & \multicolumn{4}{|c|}{1.27} \\
\hline
\end{tabular}

Pathogenicity tests of common fungi occurring with grain discoloration:

Data in Table (9) indicated that the occurrence of stored fungi increased with increasing of grain moisture content in all cultivars. Alternaria, Bipolaris and Fusarium spp were the most common fungi prior to storage, while Aspergillus and Penicillium were the most common fungi after storage for 16 months. Christensen (1969) \& Mallick and Nandi (1979) reported that at higher moisture content, the population of storage fungi, mainly species of Aspergillus and Penicillium increased with increasing period and accompanying decrease in the field fungi.

Data in Table (10) revaled that Bipolaris oryzae resulted in the highest discolored grain (58.6\%) followed by Alternaria padwickii and Curvularia lunata (51.3 and $48.2 \%$, respectively). The nun-specific fungus Bipolaris hawaiiensis caused low discolored grains (14.3\%). spergillus spp, Penicillium spp and the untreated pots (treated by distilled water) had the lowest discolored grains (2.2, 1.9 and $1.50 \%$ respectively). Dash and Narain (1988) inoculated five rice cultivars with fungal spore suspension i.e. 
El-Wahsh, S. M. et al. 
Alternaria alternata, Curvularia lunata, Fusarium and Sporothrix sp at panicle emergence. They observed that the highest percentage of discolored grain was caused by Curvularia lunata, which ranged between 51 to $56.5 \%$, while the newly detected fungus, Sporothrix spp ranged from 13.8 to $17.5 \%$. Also, Lee et al (1986) and Islam et al (2000) reported that Alternaria padwickii was the highest occurring fungus ranging from 1.33 to $44 \%$ and ranged from 1.0 to $36.6 \%$ in two tested rice cultivars. The other pathogens such as Curvularia sp., Fusarium moniliforme, Sarocladium oryzae and Bipolaris oryzae were also present on seeds and can cause grain discoloration. Curvularia lunata and Bipolaris oryzae had the highest percentage of unfilled grains as 30 and $29.7 \%$, respectively followed by Alternaria padwickii and A. alternate as 22.8 and 22.1. Lee et al (1986) mentioned that the discolored grains and unfilled grains differed according to invasion by fungi.

Table (10): Percentage of discolored and unfilled grains induced by artificial inoculation with different fungi on Giza 177 rice cultivar in the early heading stag in greenhouse conditions.

\begin{tabular}{|l|c|c|}
\hline \multicolumn{1}{|c|}{ Isolate } & Discolored grain (\%) & Unfilled grain (\%) \\
\hline Bipolaris oryzae & 58.60 & 29.70 \\
\hline Bipolaris hawaiiensis & 14.30 & 3.30 \\
\hline Alternaria alternata & 40.80 & 22.10 \\
\hline Alternaria padwickii & 51.30 & 22.80 \\
\hline Curvularia Iunata & 48.20 & 30.00 \\
\hline Nigrospora oryzae & 25.10 & 9.70 \\
\hline Sarocladiun oryzae & 19.30 & 4.50 \\
\hline Fusariun spp & 20.20 & 11.00 \\
\hline Fusarium moniliforme & 34.10 & 15.00 \\
\hline Aspergillus spp & 2.20 & 1.00 \\
\hline Penicillium spp & 1.90 & 0.90 \\
\hline Control (distilled water) & 1.50 & 0.80 \\
\hline L.S.D 5\% & 1.67 & 1.55 \\
\hline
\end{tabular}

\section{REFERENCES}

Bastiaans, L. (1993). Effects of leaf blast on photosynthesis of rice.1- Leaf photosynthesis. Netherlands Journal of plant pathology., 99: 197-203.

Christensen, C. M. (1969). Influence of moisture content. Temperature and time of storage upon invasion of rough rice by storage fungi. Phytopathology. 59: 145-148.

Daniels, M.J.; B.P. Marks; T. J. Siebenmorgen; R. W. Mcnew and J. F. Meullent (1988). Effect of long-grain rough rice storage history on enduse quality. Journal of Food Science. 63: 832-835.

Das, A.N. and A. Narain (1988). Detection of grain discoloring of fungal organisms of rice and production of disease- free seeds. Indian J. Mycol. Pl. Pathol. 18: 24-30.

El-Wahsh, S.M. (1997). Studies on both brown spot and blast diseases of rice in Egypt. Ph.D. Thesis, Faculty of Agriculture, Tanta university, Egypt. 


\section{El-Wahsh, S. M. et al.}

Fujisaka, S., R.A.Guino; R.T. Lubigan and K. Moody (1993). Farmers' Rice Seed Management Practices and Resulting Weed Seed Contamination in the Philippines. Seed Sci. Technol. 21: 149-157.

International Rice Research Institute IRRI (1997). Rice Alman ac (2nd ed.). Los Bajnos, Philippines: International Rice Research Institute.

Islam, M. Sh.; Q. S. A. Jahan; K. Bunnarith; S. Viangkum and S.D. Merca (2000). Evaluation of seed health of some rice varieties under different conditions. Bot. Bull. Acad. Sin. 41: 293-297.

ISTA. (2004). International Rules for Seed Testing. International Seed Testing Association. Seed Science and Technology.

Khanzada, A. K., and J. Khan (1987). Efficacy of some detection methods for seed borne fungi in seed health testing programmer. Pakistan $\mathrm{J}$. Agric.8 (2):231-235.

Lee, S.C.; M.E. Alvenda; J.M. Bonman and E.A. Heinrichs (1986). Insects and pathogens associated with rice grain discoloration and their relationship in the Philippines. Korean-Journal-of-Plant-Protection. 25(2): 107-112.

Macedo, E.D.C.; D. Groth; J. Soave; E. de-C-Macedo (2002). Influence of bag types on health quality of stored rice seeds. Revista-Brasileira-deSementes. 24(1): 42-50.

Mallick, A. K. and B. Nandi (1979). Role of moisture content in deterioration of rough rice in storage. Seed Sci.\& Technol. 7: 423-429.

Mia, M. and K. M. Safeeulla (1998). Survival of seed-borne inoculums of Bipolaris oryzae, the causal agent of brown spot disease of rice. Abstract of Seed - Research. 26(1):78-82.

Misra, A.K.; V. Dharam and D. Vir (1992). Effect of different agronomic practices on incidence of seed discoloration in paddy. Indian $\mathrm{J}$. Mycology and Plant Pathology. 22(1): 44-48.

Muangkaeo, R.; S. Srichuwong and S. Vearasilp (2005). Influence of Packaging Materials and Storage Time on Seed Viability and Chemical Component of Rice Seed Conference on International Agricultural Research for Development Stuttgart-Hohenheim, October 11-13, 2005.

Pearce, M.D., B.P. Marks and J.F. Meullenet (2001). Effects of post harvest parameters on functional changes during rough rice storage. Cereal Chemistry, 78: 354-357.

Pinciroli, M.; M.N. Sisterna ; R. Bezus and A. A. Vidal (2004). Rice grain discoloration: effect of nitrogen fertilization. Rev. Fac. Agron. 105 (2): 88-96.

Rahman, M., and M. Mat (1998). Studies on the health status of farmers' stored seed of rice. Bangladesh-Journal-of-Plant-Pathology. 14( 1-2): 37-40.

Rao, V. K. (1992). Impact of storage systems on grain microflora, quality and viability of baddy storage. Indian Phytopath. 4 (1): 44-48.

Seshu, D.V.; V. Krishnasamy and S.B. Siddique (1988). Seed vigour in rice. In Rice Seed Health. International Rice Research Institute, Philippines, pp. 315-329. 
Shatta, M.N.; and E.A. Salem (1999). Effect of different grain moisture contents at harvest on seed quality and invasion by storage fungi in three newly released rice cultivars. Egypt. J.Appl. Sci.,14 (17):428-442.

Sunaina, W.; C.S. Reddy and S. Waghray (1988). Seed mycoflora and aflatoxin production in rice. Indian Phytopathology . 41(3): 492-494.

تأثير فترات التخزين علي فطريات تلون الحبوب باختلاف رطوبة الحبة ومستويات التسميا

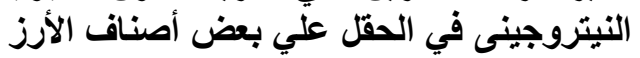

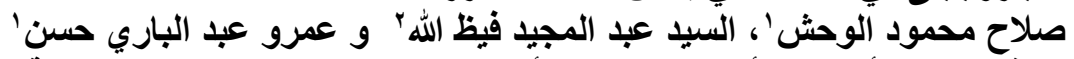

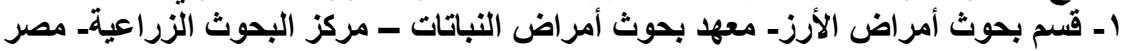

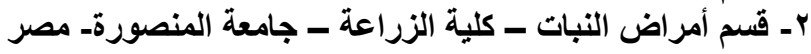

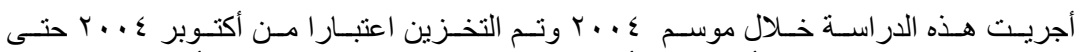

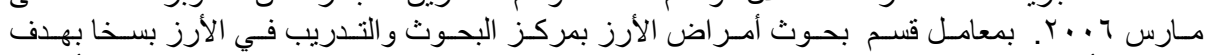

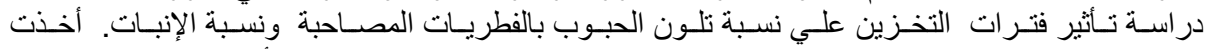

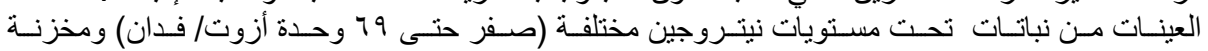

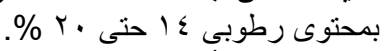

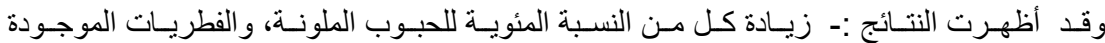

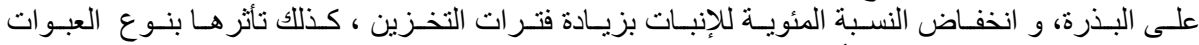

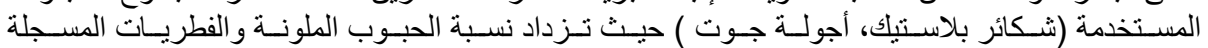

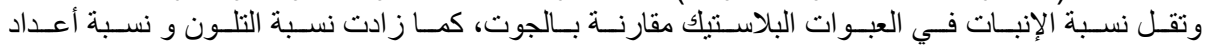

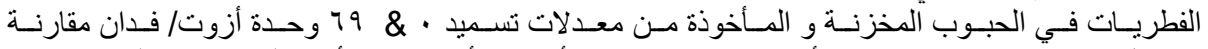

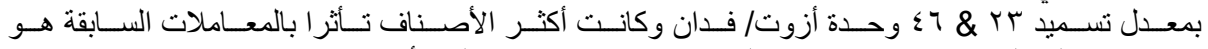

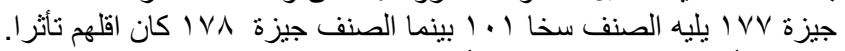

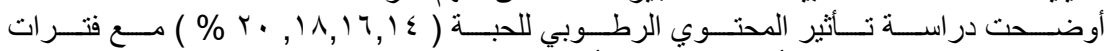

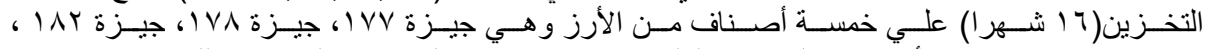

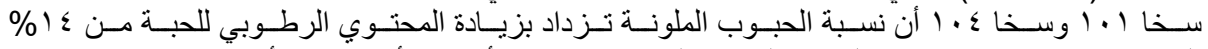

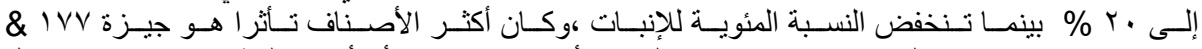

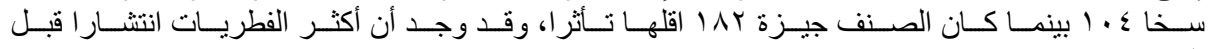

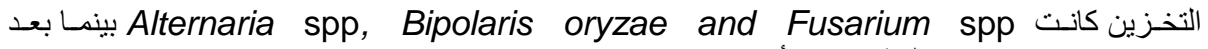
Aspergillus spp and Penicillium مرور 17 شهرا كانت الفطريـات الأكثر انتشـارا هـى

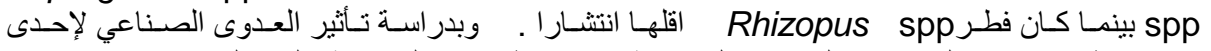

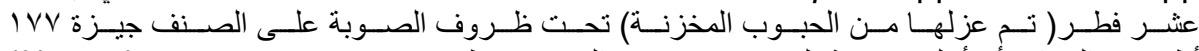

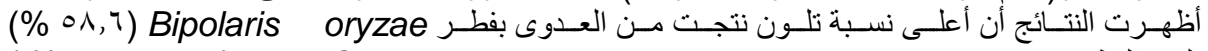

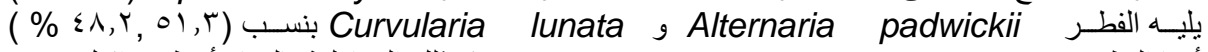
أمـا الفطر Penicillium spp و Aspergillus spp وكذلك المعاملـة بالمـاء أعطت اقل نسـب

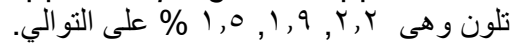



J. Agric. Sci. Mansoura Univ., 33 (3): 1997 - 2013, 2008

1871 
El-Wahsh, S. M. et al.

1872 
Table (1): Percentage of discolored grains of rice cultivars fertilized with different doses of nitrogen as influenced by storage period and type of storage bags.

\begin{tabular}{|c|c|c|c|c|c|c|c|c|c|c|c|c|c|}
\hline \multirow{3}{*}{$\begin{array}{c}\text { Storage } \\
\text { period } \\
\text { (month) } \\
\text { (P) }\end{array}$} & \multirow{3}{*}{$\begin{array}{c}\text { Nitrogen } \\
(\mathbf{k g} / \mathrm{fed}) \\
(\mathrm{N})\end{array}$} & \multicolumn{4}{|c|}{ Giza 177} & \multicolumn{4}{|c|}{ Giza 178} & \multicolumn{4}{|c|}{ Sakha 101} \\
\hline & & \multicolumn{2}{|c|}{ Kafr El-Sheikh } & \multicolumn{2}{|c|}{ EI-Dakahlia } & \multicolumn{2}{|c|}{ Kafr El-Sheikh } & \multicolumn{2}{|c|}{ EI-Dakahlia } & \multicolumn{2}{|c|}{ Kafr El-Sheikh } & \multicolumn{2}{|c|}{ EI-Dakahlia } \\
\hline & & $\begin{array}{l}\text { Striped } \\
\text { plastic }\end{array}$ & $\begin{array}{l}\text { Jute } \\
\text { sacks }\end{array}$ & $\begin{array}{l}\text { Striped } \\
\text { plastic }\end{array}$ & $\begin{array}{c}\text { Jute } \\
\text { sacks }\end{array}$ & $\begin{array}{l}\text { Striped } \\
\text { plastic }\end{array}$ & $\begin{array}{l}\text { Jute } \\
\text { sacks }\end{array}$ & $\begin{array}{l}\text { Striped } \\
\text { plastic }\end{array}$ & $\begin{array}{l}\text { Jute } \\
\text { sacks }\end{array}$ & $\begin{array}{l}\text { Striped } \\
\text { plastic }\end{array}$ & $\begin{array}{c}\text { Jute } \\
\text { sacks }\end{array}$ & $\begin{array}{l}\text { Striped } \\
\text { plastic }\end{array}$ & $\begin{array}{c}\text { Jute } \\
\text { sacks }\end{array}$ \\
\hline \multirow{4}{*}{$0^{*}$} & . & $\varepsilon 1,7$ & $\varepsilon 1,7$ & $r Y, T$ & $r r, T$ & $1 \cdot, \cdot$ & $1 \cdot, \cdot$ & $1 \varepsilon$, & $1 \varepsilon$, & $r r_{,}$ & $r r_{,}$ & $r, r$ & $r r, 1$ \\
\hline & Tr & 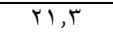 & $r, r$ & $1 \wedge, 7$ & $1 \Lambda, 7$ & $v, 1$ & 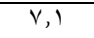 & 9,4 & $9, r$ & $T \Gamma, \Gamma$ & $1 \Gamma, \cdot$ & $T \varepsilon, T$ & $r_{0,1}$ \\
\hline & $\leqslant 7$ & $17,$. & $17,$. & $1 V, T$ & $1 Y, T$ & 7,0 & 7,0 & $\Lambda, \uparrow$ & $\Lambda, 4$ & 9,7 & 9,7 & $r \mu, r$ & $r \mu, r$ \\
\hline & 79 & $\{r, \uparrow$ & $\{r, \uparrow$ & $T \varepsilon, r$ & $r \varepsilon, \varepsilon$ & $11, \cdot$ & $11, \cdot$ & $1 \Lambda$, & $1 \Lambda$, & Tr,. & $r r, \cdot$ & $r v, \cdot$ & $r \Lambda, \varepsilon$ \\
\hline \multirow{4}{*}{1} & . & $\varepsilon r, 1$ & $\varepsilon r, \cdot$ & $r T, Y$ & $r T, O$ & $11, r$ & $1 \cdot, 1$ & 10,1 & 10,1 & $r T, \cdot$ & YY,Y & $r 1,9$ & $r 1,9$ \\
\hline & rt & $r+, 1$ & Tr, & 19,9 & $19, \mathrm{~V}$ & $9, Y$ & $\Lambda, \cdot$ & $11, \cdot$ & $11, \cdot$ & $11, \mathrm{~V}$ & $15, \lambda$ & $r 0, \Lambda$ & $T \varepsilon_{,}$ \\
\hline & $\leqslant 4$ & $1 Y, 9$ & 17,1 & $1 \wedge, \Lambda$ & $1 \Lambda, r$ & $\gamma, \xi$ & $\frac{v, 1}{2}$ & 9,1 & 9,1 & $1 \cdot, 1$ & 9,1 & $r \varepsilon, 1$ & $r \varepsilon, \cdot$ \\
\hline & 79 & $\varepsilon \varepsilon, \cdot$ & $\varepsilon r, \xi$ & $r \uparrow, \Lambda$ & rฯ, & $I r, \Lambda$ & $11, \varepsilon$ & 19,1 & $19, Y$ & $r \mu, q$ & Yr,Y & $r \Lambda, \xi$ & $r v, r$ \\
\hline \multirow{4}{*}{ ir } & - & $\varepsilon 0,1$ & $\varepsilon r,$. & $r \Lambda, r$ & $r q, Y$ & $T V, 1$ & 17,4 & $T V, \Lambda$ & $T V,$. & $r \uparrow, \Lambda$ & $T \varepsilon, Y$ & $r V, \Lambda$ & $r \varepsilon, \Gamma$ \\
\hline & rr & $r \varepsilon, \cdot$ & $r, Y, Y$ & $r r, q$ & $r \cdot, \xi$ & $1 \xi, 7$ & 11,1 & $1 \xi, r$ & $T^{\prime}, \cdot$ & 17,1 & 15,7 & $r q, 1$ & $r_{0, Y}$ \\
\hline & $\leqslant 4$ & 19,1 & $1 \Lambda$, & Yr, r & 19,0 & $I r, Y$ & $11, r$ & $1 \%, 9$ & $1 \cdot, 0$ & $1 \varepsilon, Y$ & $11, \cdot$ & $r 4,0$ & ro, \\
\hline & 79 & $\varepsilon v, \Lambda$ & $\varepsilon 0,$. & $r 1, \cdot$ & $r 9,1$ & $T V, \lambda$ & $1 \varepsilon, 1$ & $r 1,9$ & 19,9 & $r V, 1$ & $r \Psi, V$ & $\varepsilon r, \Lambda$ & $r 9,1$ \\
\hline \multirow{4}{*}{11} & $\cdot$ & $\{\wedge, \xi$ & $\{0,1$ & $r 1,1$ & $r A, r$ & 19,1 & $T, Y$ & $r \cdot, \cdot$ & $1 \wedge, \cdot$ & $r \wedge, \cdot$ & $r_{0}, \cdot$ & $\varepsilon \cdot$, & $r \bar{r}, \mathrm{r}$ \\
\hline & Yr & $r q, r$ & $r+, 1$ & $T_{0},$. & $r r, \Lambda$ & $17,$. & $1 \%, 1$ & $17,$. & $1 \%, \cdot$ & $1 \Lambda$, & 17,1 & $r \cdot, 0$ & $r q, 1$ \\
\hline & $\sum 4$ & $r, 1$ & 19,1 & $r \varepsilon, 1$ & $r 1,1$ & $17,$. & $T r, \cdot$ & 17, & $T r, r$ & $17,$. & $10,$. & $T \varepsilon, Y$ & TY,. \\
\hline & 79 & Or, $\varepsilon$ & $\varepsilon \wedge, 1$ & rT, & 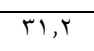 & $r \cdot, \cdot$ & 17, & $r \varepsilon, \cdot$ & $r, \cdot$ & $r q, \Lambda$ & $r V, r$ & $\{0$, & $\varepsilon \cdot$, \\
\hline \multicolumn{2}{|c|}{$\begin{array}{l}\text { L.S.D. } 5 \% \\
2-M^{* *} \text { at each } \mathrm{P} \times \mathrm{N}\end{array}$} & \multicolumn{2}{|c|}{1.40} & \multicolumn{2}{|c|}{1.76} & \multicolumn{2}{|c|}{1.65} & \multicolumn{2}{|c|}{1.63} & \multicolumn{2}{|c|}{1.64} & \multicolumn{2}{|c|}{2.47} \\
\hline
\end{tabular}

* Prior to storage.

* Prior to storage.
${ }^{\star *}$ Type of bag storage 
Table (2): Percentage of germination of rice cultivars fertilized with different doses of nitrogen as influenced by storage period and type of storage bags.

\begin{tabular}{|c|c|c|c|c|c|c|c|c|c|c|c|c|c|}
\hline \multirow{3}{*}{$\begin{array}{c}\text { Storage } \\
\text { period } \\
\text { (month) } \\
\text { (P) }\end{array}$} & \multirow{3}{*}{$\begin{array}{c}\text { Nitrogen } \\
\text { (kg/fed) } \\
\text { (N) }\end{array}$} & \multicolumn{4}{|c|}{ Giza 177} & \multicolumn{4}{|c|}{ Giza 178} & \multicolumn{4}{|c|}{$\begin{array}{l}\text { Sakha } 101 \\
\end{array}$} \\
\hline & & \multicolumn{2}{|c|}{ Kafr El-Sheikh } & \multicolumn{2}{|c|}{ El-Dakahlia } & \multicolumn{2}{|c|}{ Kafr El-Sheikh } & \multicolumn{2}{|c|}{ El-Dakahlia } & \multicolumn{2}{|c|}{ Kafr El-Sheikh } & \multicolumn{2}{|c|}{ El-Dakahlia } \\
\hline & & $\begin{array}{l}\text { Striped } \\
\text { plastic }\end{array}$ & $\begin{array}{l}\text { Jute } \\
\text { sacks }\end{array}$ & $\begin{array}{l}\text { Striped } \\
\text { plastic }\end{array}$ & $\begin{array}{l}\text { Jute } \\
\text { sacks }\end{array}$ & $\begin{array}{l}\text { Striped } \\
\text { plastic }\end{array}$ & $\begin{array}{l}\text { Jute } \\
\text { sacks }\end{array}$ & $\begin{array}{l}\text { Striped } \\
\text { plastic }\end{array}$ & $\begin{array}{l}\text { Jute } \\
\text { sacks }\end{array}$ & $\begin{array}{l}\text { Striped } \\
\text { plastic }\end{array}$ & $\begin{array}{l}\text { Jute } \\
\text { sacks }\end{array}$ & $\begin{array}{l}\text { Striped } \\
\text { plastic }\end{array}$ & $\begin{array}{l}\text { Jute } \\
\text { sacks }\end{array}$ \\
\hline \multirow{4}{*}{$0^{*}$} & $\cdot$ & 97, & $97, \cdot$ & $9 \vee$, & $9 V, \cdot$ & $9 \wedge,$. & $9 \wedge$, & 98.0 & 98.0 & $9 Y$, & $9 \vee, \cdot$ & $9 \vee$, & $9 \vee$, \\
\hline & rT & $97,$. & $97,$. & $9 \mathrm{~V}, \cdot$. & $9 \mathrm{Y},$. & $9 \Lambda,$. & $9 \Lambda, \cdot$. & 98.0 & 98.0 & $9 \mathrm{Y},$. & $9 \mathrm{~V}, \cdot$. & $9 Y, \cdot$ & $9 \mathrm{~V}, \cdot$. \\
\hline & $\leqslant \uparrow$ & $97,$. & $97,$. & $9 \vee,$. & $9 \mathrm{Y}, \mathrm{.}$ & $9 \Lambda,$. & $9 \Lambda,$. & 98.0 & 98.0 & $9 y,$. & $9 \vee, \cdot$. & $9 \mathrm{Y}, \mathrm{.}$ & $9 \mathrm{~V}, \mathrm{.}$ \\
\hline & 79 & $97,$. & $97, \cdot$ & $9 \vee,$. & $9 y, \cdot$ & $9 \Lambda,$. & $9 \wedge,$. & 97.6 & 98.0 & $9 Y, \cdot$ & $9 \mathrm{Y}, \cdot$ & $9 Y, \cdot$ & $9 \vee, \cdot$ \\
\hline \multirow{4}{*}{1} & . & $91,$. & 91,1 & $91, \mathrm{r}$ & $94, \cdot$ & $9 \Gamma, \cdot$ & $94,$. & 93.0 & 93.4 & $94, \cdot$ & $94, \cdot$ & $91, \cdot$ & $9 T_{,} \cdot$ \\
\hline & rr & $94, \cdot$ & $9 \Gamma, \cdot$ & $9 \Gamma,$. & $9 \%, \cdot$ & 9 q६, & 9 q६, & 94.0 & 94.2 & $9 r, \cdot$ & $9 \mathrm{qr},$. & $9 \%, \cdot$ & 94,0 \\
\hline & $\leqslant \uparrow$ & $9 T, \Lambda$ & $9 \Gamma,$. & $9 T,$. & $9 \%,$. & $9 \xi,$. & $9 \varepsilon,$. & 94.0 & 94.6 & $9 \%,$. & $94, \mathrm{r}$ & $9 \%,$. & $9 \pi,$. \\
\hline & 79 & $91, \varepsilon$ & $94,$. & $94, \cdot$ & $9 q_{,} \cdot$ & $94, r$ & $9 \pi,$. & 93.0 & 91.8 & $9 T,$. & $9 T, Y$ & $9 \mathrm{qr},$. & $9 T, r$ \\
\hline \multirow{4}{*}{ ir } & $\cdot$ & $\Lambda 4$, & $\Lambda \Lambda$, & $\Delta \vee$, & $\Lambda \Lambda$, & $\wedge 9,$. & $\wedge 9,$. & 88.6 & 89.0 & $\Lambda \Lambda$, & $\wedge 9, \cdot$ & $\Lambda \uparrow$, & $\mathrm{AV}, \cdot$ \\
\hline & $T r$ & $\lambda 9,$. & $\Lambda 9, \cdot$ & $q_{\cdot, \cdot}$ & $q_{\bullet \cdot, \cdot}$ & 91,0 & 91,0 & 90.0 & 90.0 & $9 \cdot, \cdot$ & $9 \cdot, r$ & $\Lambda 9,$. & $19, \cdot$. \\
\hline & $\leqslant \uparrow$ & $\Lambda_{\Lambda,}$ & $\wedge 9,$. & $9 \cdot, \cdot$ & $q_{\bullet, .,}$ & 91,0 & 91, . & 91.0 & 91.2 & $q_{\bullet, .,}$ & $9 \cdot, \cdot$ & $\Lambda \Lambda$, & $\wedge 9,$. \\
\hline & 79 & $\Lambda 4$, & $\lambda V^{\prime}$, & $\wedge \uparrow$, & $\Lambda V$, & $\Lambda \Lambda$, & $\Lambda \wedge$, & 87.3 & 84.0 & $\lambda V^{\prime}$, & $\Lambda \Lambda$, & $10, \cdot$ & $\Lambda 4$, \\
\hline \multirow{4}{*}{11} &. & $74,$. & $v_{1, \cdot}$ & $V_{\bullet}, \cdot$ & $V r, \cdot$ & $V \wedge, \cdot$ & $\lambda \cdot, \varepsilon$ & 76.3 & 79.0 & $V \Psi, \cdot$ & $V \varepsilon \varepsilon$, & 67.0 & $V_{Y, \cdot}$ \\
\hline & YT & \rceil$\Lambda, \xi$ & $V Y, \cdot$ & $V Y, I$ & $V_{0, Y}$ & $v 9,$. & 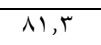 & 77.0 & 80.6 & $V \varepsilon, \cdot$ & $V 4, \cdot$ & $79, r$ & $V \varepsilon, T$ \\
\hline & $\sum \uparrow$ & $7 \Lambda,$. & $\overline{V Y, \cdot}$ & $V Y, \cdot$ & $v_{0},$. & $v_{9},$. & $\Lambda 1,$. & 77.0 & 80.0 & $V \xi \varepsilon$, & $V{ }^{4},$. & 79,6 & $V \varepsilon,$. \\
\hline & 79 & $7 \xi, 1$ & $T V, T$ & $T V, 0$ & $V I, Y$ & $V_{0},$. & $V \wedge,$. & 74.0 & 78.0 & $79,$. & $V_{Y,}$ & $70,$. & 79,1 \\
\hline \multicolumn{2}{|c|}{$\begin{array}{l}\text { S.D. } 5 \% \\
2-M^{* *} \text { at each } P \times N\end{array}$} & \multicolumn{2}{|c|}{1.54} & \multicolumn{2}{|c|}{1.44} & \multicolumn{2}{|c|}{1.44} & \multicolumn{2}{|c|}{2.86} & \multicolumn{2}{|c|}{1.48} & \multicolumn{2}{|c|}{1.22} \\
\hline
\end{tabular}

${ }^{\star \star}$ Type of bag storage

* Prior to storage. 
Table(9): Effect of moisture content of rice grain on fungi occurrence in different rice cultivars before and after storage.

\begin{tabular}{|c|c|c|c|c|c|c|c|c|c|c|c|c|c|c|c|c|c|}
\hline \multirow[b]{2}{*}{ Cultivar } & \multirow[b]{2}{*}{$\begin{array}{l}\text { Moisture } \\
\text { content } \%\end{array}$} & \multicolumn{8}{|c|}{ Prior to storage } & \multicolumn{8}{|c|}{16 months after storage } \\
\hline & & $\begin{array}{l}\frac{9}{2} \\
\frac{\pi}{0} \\
\frac{\pi}{0} \\
\frac{2}{0}\end{array}$ & 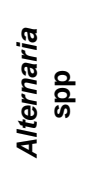 & 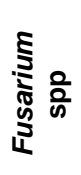 & 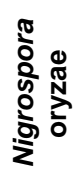 & 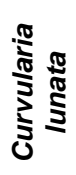 & 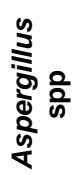 & 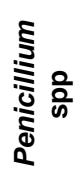 & 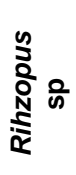 & $\begin{array}{l}\frac{n}{2} \\
\frac{\pi}{\pi} \\
\frac{2}{2} \\
\frac{2}{0}\end{array}$ & 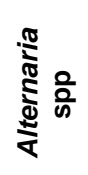 & 荵 & 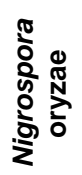 & $\underbrace{\frac{\pi}{\pi}}_{0} \frac{\pi}{\frac{\pi}{5}}$ & $\begin{array}{l}\frac{9}{3} \\
\frac{5}{8} \\
\frac{\delta}{2} \\
\frac{\delta}{2}\end{array}$ & 志 & 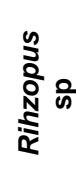 \\
\hline \multirow{4}{*}{ Giza 177} & 14 & 10.0 & 20.0 & 8.0 & 4.0 & 3.0 & 3.0 & 2.0 & 0.0 & 4.0 & 28.1 & 11.0 & 6.0 & 4.0 & 40.0 & 30.0 & 0.0 \\
\hline & 16 & 11.0 & 21.0 & 8.0 & 4.0 & 3.0 & 4.0 & 2.0 & 0.0 & 0.2 & 30.0 & 30.0 & 6.0 & 4.5 & 55.0 & 55.0 & 5.0 \\
\hline & 18 & 12.1 & 22.3 & 10.2 & 5.2 & 3.5 & 5.0 & 4.0 & 0.0 & 0.0 & 41.0 & 85.0 & 9.5 & 8.5 & 94.0 & 85.0 & 30.0 \\
\hline & 20 & 12.2 & 22.3 & 11.0 & 6.1 & 3.5 & 5.0 & 5.0 & 0.0 & 0.0 & 48.0 & 90.0 & 9.9 & 9.7 & 95.0 & 89.0 & 43.0 \\
\hline \multirow{4}{*}{ Giza 178} & 14 & 6.0 & 13.0 & 6.3 & 4.0 & 1.1 & 2.0 & 0.0 & 0.0 & 1.0 & 27.0 & 9.9 & 6.0 & 6.3 & 30.0 & 25.0 & 0.0 \\
\hline & 16 & 6.0 & 13.5 & 7.0 & 4.3 & 1.5 & 2.1 & 1.0 & 0.0 & 0.0 & 30.0 & 28.0 & 6.0 & 6.5 & 48.0 & 50.0 & 3.0 \\
\hline & 18 & 7.1 & 14.1 & 7.5 & 5.0 & 20 & 3.0 & 20 & 1.0 & 0.0 & 36.0 & 73.0 & 8.0 & 7.0 & 920 & 88.0 & 18.0 \\
\hline & 20 & 74 & 142 & 8.0 & 5.0 & 20 & 32 & 20 & 1.0 & 0.0 & 41.0 & 77.0 & 8.0 & 7.3 & 920 & 88.0 & 23.0 \\
\hline \multirow{4}{*}{ Sakha 101} & 14 & 7.0 & 14.1 & 7.1 & 3.0 & 2.0 & 2.0 & 2.4 & 0.0 & 2.5 & 28.0 & 9.8 & 6.3 & 3.0 & 38.0 & 28.0 & 0.0 \\
\hline & 16 & 7.5 & 14.8 & 7.4 & 3.5 & 2.0 & 2.0 & 2.0 & 0.0 & 0.0 & 30.0 & 30.0 & 6.3 & 3.2 & 50.0 & 48.0 & 3.0 \\
\hline & 18 & 8.4 & 17.1 & 8.0 & 4.0 & 2.6 & 4.0 & 3.1 & 1.0 & 0.0 & 38.0 & 75.0 & 11.1 & 8.0 & 91.0 & 89.0 & 17.0 \\
\hline & 20 & 8.6 & 17.4 & 8.1 & 4.0 & 2.9 & 4.0 & 4.0 & 1.0 & 0.0 & 44.0 & 81.0 & 12.1 & 8.9 & 92.0 & 90.0 & 22.0 \\
\hline \multirow{4}{*}{ Sakha 104} & 14 & 10.0 & 18.0 & 7.8 & 3.8 & 3.0 & 2.0 & 2.0 & 0.0 & 5.0 & 30.0 & 12.0 & 5.8 & 4.0 & 45.0 & 35.0 & 0.0 \\
\hline & 16 & 10.6 & 19.0 & 8.1 & 3.8 & 3.1 & 2.8 & 2.0 & 0.0 & 0.0 & 36.0 & 35.0 & 6.2 & 6.0 & 60.0 & 55.0 & 5.0 \\
\hline & 18 & 10.9 & 20.3 & 9.1 & 4.0 & 3.2 & 3.4 & 3.1 & 1.0 & 0.0 & 48.0 & 85.0 & 9.1 & 9.3 & 93.0 & 90.0 & 32.0 \\
\hline & 20 & 10.9 & 20.4 & 9.2 & 4.2 & 3.3 & 3.6 & 4.0 & 1.0 & 0.0 & 50.0 & 91.8 & 12.0 & 10.2 & 95.0 & 90.0 & 45.0 \\
\hline \multirow{4}{*}{ Giza 182} & 14 & 1.0 & 6.0 & 3.8 & 1.9 & 1.1 & 0.0 & 0.0 & 0.0 & 0.0 & 10.0 & 10.1 & 3.0 & 3.0 & 10.0 & 13.0 & 0.0 \\
\hline & 16 & 1.0 & 7.0 & 3.8 & 2.0 & 1.2 & 0.0 & 0.0 & 0.0 & 0.0 & 12.1 & 25.1 & 3.0 & 3.5 & 13.0 & 45.1 & 0.0 \\
\hline & 18 & 0.0 & 7.0 & 5.0 & 2.0 & 2.0 & 0.0 & 1.0 & 0.0 & 0.0 & 20.1 & 65.0 & 5.1 & 6.0 & 64.1 & 50.8 & 40.3 \\
\hline & 20 & 0.0 & 8.0 & 5.3 & 2.3 & 2.0 & 2.0 & 1.0 & 0.0 & 0.0 & 21.9 & 69.1 & 5.3 & 6.6 & 65.1 & 85.3 & 45.1 \\
\hline \multicolumn{2}{|c|}{ L.S.D 5\% } & \multicolumn{8}{|c|}{0.88} & \multicolumn{8}{|c|}{0.99} \\
\hline
\end{tabular}


J. Agric. Sci. Mansoura Univ., 33 (3): 1997 - 2013, 2008 
J. Agric. Sci. Mansoura Univ., 33 (3), March, 2008

1997

1998

1999

2000

2001

2002

2003

2005

2004

2005

2006

2007

2008

2009

2010

2011

2012

2013 